\title{
Suitability of the wireless testbed w-iLab.t for VANET research
}

\author{
(Invited Paper) \\ Wim Vandenberghe, Ingrid Moerman and Piet Demeester \\ Ghent University - IBBT \\ Department of Information Technology (INTEC) \\ Gaston Crommenlaan 8 Bus 201 \\ B-9050 Ghent, Belgium \\ Email: wim.vandenberghe@intec.ugent.be
}

\author{
Hans Cappelle \\ imec - IBBT \\ Kapeldreef 75 \\ B-3001 Leuven, Belgium \\ Email: hans.cappelle@imec.be
}

\begin{abstract}
Two quite different tools are normally used in the research of Vehicular Ad Hoc Networks (VANET). Simulators are practical and cost-efficient, but are not entirely accurate representations of the real life situation. Field operational tests (FOT) provide exactly the opposite characteristics. A wireless testbed is situated somewhere in between, but has not yet been applied in VANET research. In this paper it is researched if such a wireless testbed could be suitable for this kind of research. The corresponding requirements are defined, and it is investigated how the generic indoor wireless testbed w-iLab.t can be made compliant using only software adjustments. Proposed techniques are an approximation of the IEEE 802.11p standard using .11a hardware, the emulation of mobility based on link impairment, and the use of low transmit power together with manual topology configuration. These techniques are generic, and can be applied on other wireless testbeds. However, some limitations have to be taken into account. For the highway setting, w-iLab.t can provide densities up to $25 \%$ of the actual maximum VANET density in intense but flowing traffic. Experiments representing urban scenarios are labour intense and limited in topology size. Based on these observations, it is concluded that a generic wireless testbed is a valuable but complementary tool in VANET research.
\end{abstract}

\section{INTRODUCTION}

Vehicular Ad Hoc Networks (VANET) are a relatively recent networking paradigm. These networks provide local Vehicle to Vehicle (V2V) and Vehicle to Infrastructure (V2I) communication using the specifically designed IEEE 802.11p technology. This way, numerous novel cooperative applications can be introduced that increase the "time horizon", the quality and reliability of information available to the drivers about the road conditions and other vehicles in their immediate environment. Examples of such applications are emergency electronic brake lights, road hazard warnings, approaching emergency vehicle warning, slow vehicle warning, etc [1]. Attracted by the impact of such applications, research activities in the VANET domain have increased tremendously during the last years. A common property of most existing studies is the fact that they only rely on wireless network simulators to achieve their results. The advantage of these simulators is their cost efficiency and practicability, allowing the research of scenarios with a large number of vehicles. This approach to VANET research has led to to valuable insights in the domain, e.g. the identification of the VANET scalability problem [2][6]: under high vehicle densities, the IEEE 802.11p CSMA/CA scheme cannot provide adequate communication performance in terms of delay and reliability.

Although simulation-based VANET research has already produced important results, it has to be considered that wireless simulators are not entirely correct representations of the real life situation. This is caused by several factors such as simplified propagation and interference models, inaccurate application data patterns, etc. A validation of simulation results using implementations on actual hardware (and vice versa) is necessary in VANET research. However, very few studies were implemented on such hardware, and they were typically deployed on a small scale only. Such implementations are useful as proof-of-concept demonstrations in realistic environmental circumstances. However, they cannot provide meaningful insights in the performance and reliability that can be expected if the same solution would be deployed on a realistic scale.

In this paper we aim to fill this void between simulations and hardware implementations by applying a generic indoor wireless testbed for VANET research. The goal is to provide the required techniques to enable this research without any hardware adjustments to the existing w-iLab.T wireless testbed, provided by IBBT. Such techniques can also allow other institutions to transform their generic wireless testbeds into VANET research tools, providing the research community a complementary tool besides simulation platforms and small scale proof-of-concept demonstrators.

\section{AdDed VAlue of WiREless TESTBED}

As already mentioned, several studies exist that focus on VANET performance. These are often based on wireless network simulators (e.g. NS-2, NS-3, OMNeT++ or SWANS) in combination with vehicular movement simulators such as SUMO or VISSIM [2], [4], [6], [17]. The advantage of this approach is the fact that comprehensive scenarios can be researched in very early stages of development (even before the availability of hardware prototypes) and in a very costefficient manner. From a practical viewpoint this approach to VANET research also has the advantage that experiments are 
repeatable, and parameters such as amount of VANET nodes and vehicle movement patterns can be easily controlled.

However, simulations of wireless networks are not entirely representative for the real-life performance. Several studies exist that elaborate this problem in more detail [19]-[22]. The phenomenon can be caused by several factors. The large amount of simulation parameters can easily lead to human error in the configuration of the simulation scenario. Regarding signal propagation and interference, several aspects are often simplified in wireless network simulators. For example, obstacles such as walls and building are not taking into account, the transmission area is considered to be a perfect circle (while in reality this is not the case at all), and signal strength is determined in function of distance (not taking multi-path fading and other influences into account). Cavin et al. [20] demonstrated the consequences of these simplifications by comparing the results of the same scenario in three different simulators. A large discrepancy between these results was observed. There are also networking aspects that are not simplified, but entirely discarded by simulators. An example is adjacent channel interference that can influence performance of multi-interface nodes [23]. Besides networking aspects, other elements such as traffic patterns and end-user models can also be incomplete in simulations.

Therefore, there is a need to provide complementary tools. In the VANET domain, Field Operational Tests (FOTs) are in general considered as the appropriate methodology to meet this demand. Since FOTs focus on experimental research of the developed VANET solutions on public roads with real vehicles and test users, they can accurately capture characteristics such as end-user models, hard- and software faults and signal behavior assumptions. The downside of FOTs is their practical limitation. Test are not exactly repeatable, they require extensive funding, and even with a large amount of equipped vehicles (e.g. the German simTD project [24]) it is quite hard to test scenarios with specific topology requirements, e.g. VANET saturation in dense traffic.

To meet the needs left open by the previously described research tools, a generic wireless testbed in a lab environment could be a useful instrument. Such testbeds typically consist of a large amount of wireless devices supporting different technologies such as IEEE $802.11 \mathrm{a} / \mathrm{b} / \mathrm{g} / \mathrm{h}$, IEEE 802.15 .4 or Bluetooth. In general these devices are not mobile and installed indoor in a dedicated area or in the offices of the owner organization. These devices are connected to a control infrastructure for experiment management. Some example testbeds are ORBIT, MoteLab, TWIST and IBBT w-iLab.t [25]-[28]. Although the usage of such infrastructures is quite common in research domains such as Wireless Sensor Networks (WSN), Wireless Mesh Networks (WMN) or Future Internet Research and Experimentation (FIRE), they are hardly ever used in the scope of VANETs. To the best of our knowledge, the only study that relied on this kind of testbeds for VANET research is Ramachandran [25]. This study made use of the ORBIT testbed. It was the first large-scale experimental study using an amount of 100 IEEE 802.11a nodes to evaluate many-to-

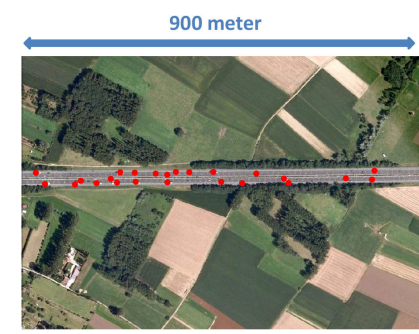

900 meter

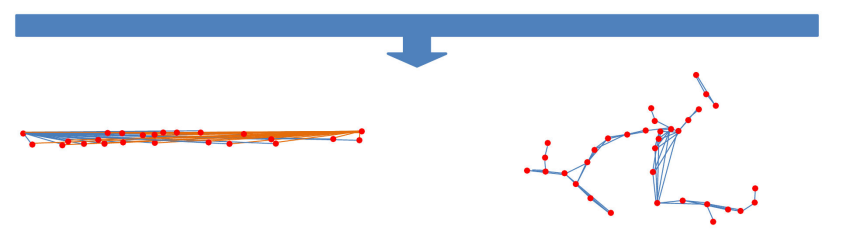

Fig. 1. Topology in highway and urban setting. In highway environment, only links starting from the upper left and upper right node were depicted to avoid overloading the picture. A correct representation would be fully meshed, with a link between every possible pair of nodes. In the urban setting however, all actual links are depicted.

many broadcast performance. However, some aspects of this study can be further improved since it not entirely captured the VANET scenario (as described in section IV).

\section{BREAKDOWN OF VANET SCENARIO}

If generic wireless testbeds are applied in VANET research, it is important that they properly represent actual VANET scenarios. In this section an overview is given of the characteristics of such a scenario.

The network topology is determined by the environment. In the highway setting, generally there are no other roads nearby, and few obstacles hinder wireless communication between vehicles. This leads to a fully meshed ribbon-like topology with high communication ranges (as depicted in Fig. 1). Some VANET aspects become less challenging in this environment (e.g. position based routing), but other problems can be greatly enhanced (e.g. the scalability problem). In urban environments, the high frequency $802.11 \mathrm{p}$ signal $(5.9 \mathrm{GHz})$ is completely blocked by buildings and other obstacles. This leads to graphlike network topologies which resemble the road topology and where the communication range between vehicles is shorter and the connectivity degree is lower (as depicted in Fig. 1). In this case position based routing becomes more challenging, and the scalability problem is reduced.

Similar to network topologies, movement speeds are also determined by the environment. This is important in the VANET context since at higher speeds the topology changes are more frequent and link quality degradation can occur. This degradation was reported by several authors, which noticed significant flooring of the packet error rate (PER) for a conventional receiver design (even in line-of-sight conditions) [7]-[9]. On highways, the relative speed difference between VANET nodes can range from $0 \mathrm{~km} / \mathrm{h}$ in case of communication between vehicles traveling at the same speed in the same direction to approximately $260 \mathrm{~km} / \mathrm{h}$ when two vehicles moving in the opposite direction communicate. In case of 
communication between a fast moving vehicle and a stationary Roadside Unit (RSU) this speed difference is about $130 \mathrm{~km} / \mathrm{h}$. In urban environments, traffic moves slower, and relative speed differences are situated between 0 and $100 \mathrm{~km} / \mathrm{h}$.

Vehicle density is an important parameter in VANET research, closely related to the scalability problem. To assess appropriate values for this parameter, a model was reused that was developed by the authors of this paper to assess the impact of road charging applications on mobile networks [10]. The model relies on actual highway vehicle traffic measurements for the Flemish region of Belgium. It can be used to determine VANET densities by changing the meaning of one model parameter. The outcome is that in flowing conditions at peak hour, densities can rise up to approximately 25 vehicles per kilometer per lane, corresponding with an inter-vehicle distance of 40 meters. Taking the $802.11 \mathrm{p}$ communication range of 1000 meters into account, the diameter of a single VANET transmission domain becomes 2000 meter, containing up to 300 network nodes in flowing conditions on a 6-lane highway (three lanes in each direction). In jammed conditions, this model cannot be applied. Instead, we rely on the analytical representation of a traffic jam defined in other previous work [11]. For the highway scenario with three lanes in both directions and a traffic jam in each direction it was estimated that the distribution of vehicles is 631 vehicles per kilometer. This leads to a single transmission domain of about 1200 vehicles. Both vehicle density values are in line with other VANET scenarios mentioned in literature [3], [12], [13].

Packet size is an important variable that can greatly influence the results of performance experiments. Almost all applications powered by VANET communications rely on two types of messages: cooperative awareness (CAM) and decentralized environment notification messages (DENM). Both message types are the subject of ETSI standardization [14], [15]. The size of a CAM message varies between approximately 25 and 50 bytes, depending on the chosen optional data fields. For DENM messages this range is 40 to 80 bytes. Without a doubt, these data messages will be accompanied by security data. As shown by Zhang et al. [16], efficient message authentication schemes can restrict the security overhead to 250 bytes per packet. This means that the length of secure CAM and DENM messages will be approximately 300 bytes. For the message generation frequency we can refer to the ETSI standard regarding cooperative applications [1], which states that the message generation frequency can be 1,2 and $10 \mathrm{~Hz}$.

\section{TESTBED REQUIREMENTS}

Based on the above description of the VANET scenario, the requirements for generic testbeds can be defined to be suitable for VANET research. First of all it most support the IEEE 802.11p technology that is envisaged for vehicular communications. This wireless hardware should also support packet sizes which correspond with CAM and DENM messages (300 bytes) under all circumstances. To support the highway scenario, it should be able to provide a large number of nodes within a single transmission domain. On the other hand, to support urban scenarios it should be possible to define multi-hop topologies in the experiments. Degradation of link quality because of the high relative speed differences between network nodes should also be taken into account.

Based on these requirements, it can be concluded that no generic wireless testbed has yet been adjusted adequately to support VANET research. As already mentioned, only the study of Ramachandran [25] used a testbed for VANET research (ORBIT), but failed to capture all aspects of the VANET scenario. The experiments relied on IEEE 802.11a technology instead of IEEE 802.11p, the effect of mobility was not considered and multi-hop communication was not supported.

\section{IMPLEMENTATION ON IBBT W-ILAB.T}

Since the requirements were determined in the previous section, it is now possible to investigated if the IBBT wiLab.t wireless testbed can be made suitable for VANET research. In this section, the testbed itself is introduced and for every requirement it is investigated if the testbed complies. If required, software techniques are proposed to ensure usability of the testbed.

\section{A. Description of IBBT w-iLab.t}

The IBBT w-iLab.t wireless lab is an extensive test facility, with a primary goal of supporting WSN and WMN research. It is introduced in detail in Bouckaert et al. [28]. The infrastructure has been rolled out on three floors $(17.5$ x 90 meter) of the IBBT office premises in Ghent, Belgium. The network consists of 200 network nodes based on hardware compatible with the Intel $\mathrm{x} 86$ architecture (PC Engines Alix boards), providing maximum flexibility to researchers and developers. Each board is equipped with two Compex WLM54SAG 200mW AR5006XS 802.11a/b/g 54/108 Mbps miniPCI wireless cards, and a Tmote Sky IEEE 802.15.4 mote. These nodes are centrally managed for control and monitoring purposes and remote access, and can be easily configured, including installation of new software, protocols, middleware components, etc.

In the context of VANET research, the installation on the second and third floor of the IBBT premises are most interesting. As can be seen in Fig. 2, 58 nodes are installed on the second floor, and 55 nodes on the third floor, both in a linear distribution which resembles the highway ribbonlike topology. On every node two completely independent VANET protocol stacks are active, one for every IEEE 802.11 interface. As a result, an experiment can consist of 110 independent VANET nodes. In practice, there is practically no communication possible between nodes on different floors due to the cross-floor signal propagation characteristics. Therefore an experiment in the context of VANET research is limited to a single floor. However, first WMN experiments revealed that it is hard to create multi-hop topologies using the IEEE 802.11 interfaces on a single floor, mainly because their transmission power cannot be set lower than $0 \mathrm{dBm}$. Therefore fixed attenuators of $10 \mathrm{~dB}$ were added to all nodes 


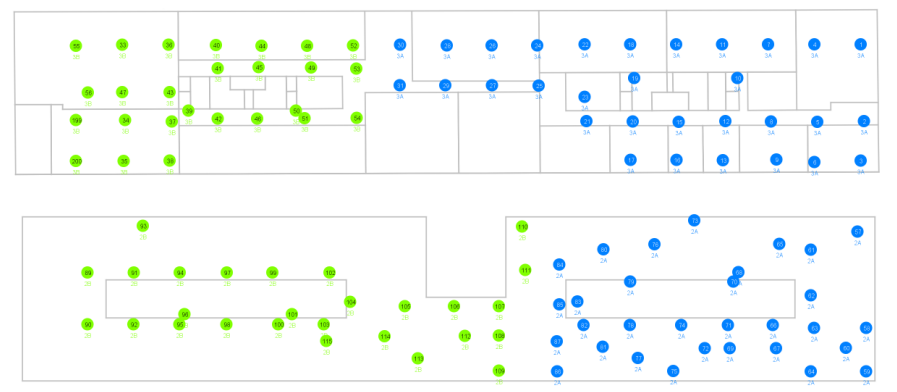

Fig. 2. Topology of w-iLab.t at the second (bottom) and third (top) floor of the IBBT office premises. The blue and green colors indicate zones that can be used independently, but they can also be combined in a single experiment.

on the third floor, and of $20 \mathrm{~dB}$ on the second floor. As a result, experimenters can vary the perceived node density from sparsely to very densely connected by configuring appropriate transmission power values and selecting the suitable floor for their experiments.

\section{B. Approximation of $802.11 p$}

As mentioned in section IV, a VANET testbed should be equipped with IEEE 802.11p wireless interfaces. However, the w-iLab.t nodes are not equipped with such interfaces, but with IEEE $802.11 \mathrm{a} / \mathrm{b} / \mathrm{g}$ wireless cards. A solution to this problem could be to replace the current cards with newer ones supporting the $.11 \mathrm{p}$ standard. At the time of writing, and to the best of our knowledge, only one IEEE $802.11 \mathrm{p}$ wireless card is already commercially available: the Unex DCMA-86P2 miniPCI card [29]. Although this solution is technically feasible, the downside of this approach is that w-iLab.t would no longer be able to support other kinds of IEEE 802.11 experiments since the frequency range of this card is limited to the 5.9 $\mathrm{GHz}$ VANET frequency band only. This is unacceptable.

However, the difference between the IEEE 802.11p amendment and the other IEEE 802.11 standards is quite small. In short, it can be seen as a combination of the IEEE 802.11a and IEEE 802.11e standards, with some specific adjustments. With this information in mind, it is possible to implement an approximation of the IEEE 802.11p standard using the standard off the shelf IEEE 802.11a hardware of w-iLab.t and some specific software adjustments. This work has already been performed and was presented in previous work [30]. It was concluded that several differences could be eliminated, other differences were approximated in such a way that the introduced deviations of the $.11 \mathrm{p}$ standard can be neglected, but some aspects however could not be approximated. The channel bandwidth remains $20 \mathrm{MHz}$ instead of the desired 10 $\mathrm{MHz}$, and the maximum allowable Effective Isotropic Radiated Power (EIRP) remains $200 \mathrm{~mW}$ instead of $2 \mathrm{~W}$. Under real vehicular circumstances this results in a less robust signal under high velocity, and in a lower communication range compared to $.11 \mathrm{p}$ hardware. This is not an insuperable obstacle in the case of w-iLab.t since all nodes are placed no further than 90 meters apart and will not actually move.

\section{High vehicle density}

As described in section III, VANET scenarios can be characterized by high vehicle densities, especially in the highway setting. This means that a large number of nodes is present in the same transmission domain, each contending for the same shared wireless channel. As indicated by the scalability problem, the IEEE 802.11p MAC scheme cannot handle such a situation efficiently. The identification of suitable optimizations to handle this problem is a very important topic within VANET research, and should certainly be supported by a VANET testbed. In the case of w-iLab.t, the installation at the third floor seems most interesting in this context, since less powerful attenuators of $10 \mathrm{dBm}$ have been installed there.

To investigate the number of nodes present in a single transmission domain, an experiment was performed on this floor. During this experiment, all nodes listen to broadcast messages for the entire duration of the test, and in a coordinated rotation each of the 110 VANET nodes transmits 100 broadcast messages. At the end of the experiment, all nodes produce a report that lists the amount of messages they received from each sender. If a node received $95 \%$ of the packets transmitted by a specific node, we define this sending node as part of the transmission domain of the receiver. This way, the size of the transmission domain of every node can be determined. The result of this experiment is depicted in Fig. 3. At maximum transmission power of $23 \mathrm{dBm}$ and $3 \mathrm{Mbps}$ data rate (BPSK 1/2), the size of the transmission domain of each node varies between 40 and 100 nodes, with an average of 75 nodes. With the same output power, $6 \mathrm{Mpbs}$ (QPSK $1 / 2$ ) results in an average transmission domain size of 53 nodes, while 12 Mbps (16QAM 1/2) results in a transmission domain with an average size of 47 nodes. It can be concluded that for scenarios with high vehicle densities, it is advised to combine the maximum transmission power of $23 \mathrm{dBm}$ with the most robust BPSK 1/2 modulation ( $3 \mathrm{Mpbs}$ ). In that case, a single transmission domain contains in average 75 nodes, which is $25 \%$ of the transmission domain corresponding with intense but flowing traffic (see section III). This indicates that a combination of testbed and simulation experiments is required in research of the VANET scalability problem. In that case, as previously proposed by Ramachandran et al. [25] the testbed can serve as a reference scenario for validation of network simulators.

\section{Multi-hop support}

To enable testing of multi-hop scenarios, it is important that the testbed can be configured in such a way that nodes can only communicate with a limited number of nearby nodes. The second floor of w-iLab.t seemed most interesting for this kind of experiments, since attenuators of $20 \mathrm{dBm}$ are installed on every wireless interface. However, a similar experiment as in the previous subsection $\mathrm{V}-\mathrm{C}$ indicated that even at the maximum transmit power of $23 \mathrm{dBm}, 42 \%$ of all nodes could not communicate with more than one neighbor. This means that all these nodes cannot be applied as intermediate nodes in a multi-hop topology. As a result, this floor is not 


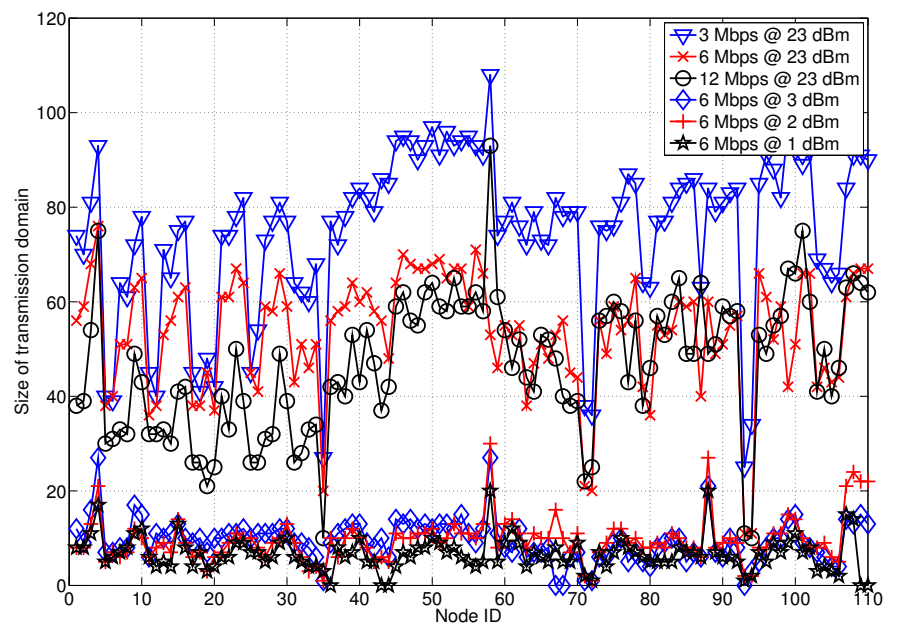

Fig. 3. Transmission domain analysis of the third floor of w-iLab.t. On this floor, attenuators of $10 \mathrm{~dB}$ where installed on every wireless interface For every node on this third floor, the amount of nodes that can successfully communicate with this node is depicted for different modulations and transmit powers. It can be concluded that by selecting the appropriate transmit power, both high-density and multi-hop scenarios can be supported.

appropriate for the execution of multi-hop scenarios in the VANET context.

However, the combination of the less stringent attenuation at the third floor together with low transmission powers results in far more favorable conditions for this kind of experiments. As depicted in Fig. 3, transmission powers of $1-3 \mathrm{dBm}$ result in smaller and quite equal transmission domains for all nodes. Because every embedded PC of the testbed powers two independent VANET nodes, this size can easily be halved by only using one of the two wireless interfaces per PC. In that case, every node can communicate in average with 4.5 neighbors at 3 and $2 \mathrm{dBm}$, and with 3 neighbors at $1 \mathrm{dBm}$. The amount of nodes with less then 2 neighbors is about $8 \%$ at 3 and $2 \mathrm{dBm}$, but $15 \%$ at $1 \mathrm{dBm}$. Based on these results, it is concluded that $2 \mathrm{dBm}$ is the most appropriate transmit power setting for multi-hop scenarios, since this value results in the smallest transmission domain without sacrifycing the ability to relay multi-hop packets. Due to the grid-like distribution of the w-iLab.t nodes on this third floor, multi-hop topologies can be created by carefully selecting which nodes should be activated during an experiment, and which not. However, this translation from a given topology to a an appropriate set of active w-iLab.t nodes is quite labour intense, and the total amount of hops in a single path is limited. This indicates that similar to the highway setting, a combination of testbeds and simulators is required for the urban environment.

\section{E. Emulation of mobility}

One of the most important downsides of using the w-iLab.t wireless testbed for VANET experimentation is the lack of actual node movement. To emulate the effects of node mobility on link quality in such indoor wireless lab experiments, we will utilize link impairment techniques. These are based on simulations of the link quality degradation induced by high

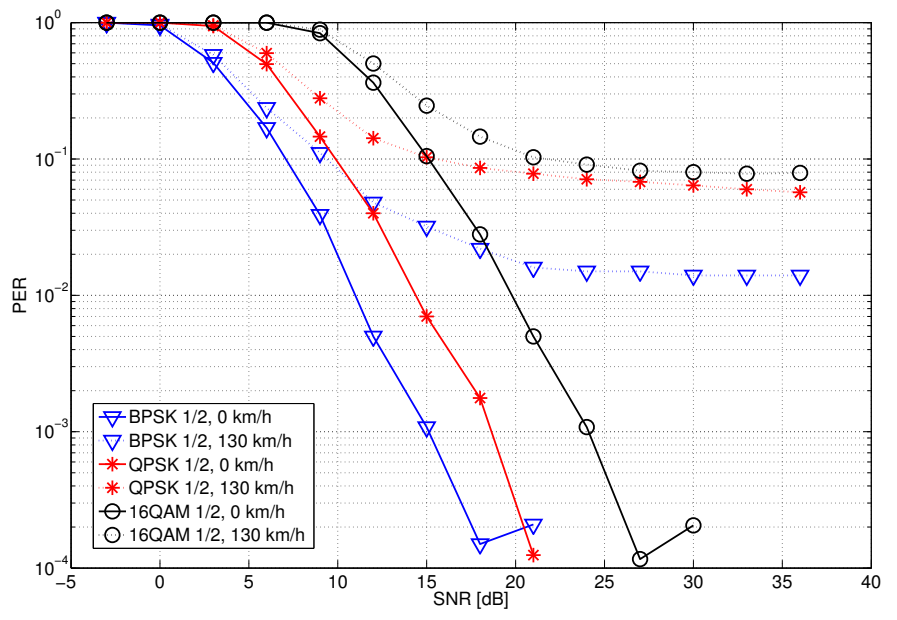

Fig. 4. Example of physical layer modeling results. Packet Error Rate is determined in function of signal-to-noise ratio, several modulations and relative speed differences are considered. The simulated environment is a highway, packet size is 300 bytes. On the figure only a few of all investigated combinations of modulation and speed are depicted. All results can be exported to lookup tables that can be used for link impairment which emulates the effect of mobility on link reliability.

relative speed differences. To capture these effects, we relied on the Matlab model that is used at imec to simulate the physical layer of the IEEE802.11p standard. This model consists out of transmitter and receiver models, a channel model and scripts for visualization and analysis. Several scenarios can be explored by means of configurable parameters, such as vehicle speed and channel conditions. Appropriate modeling of the vehicular channel conditions is essential to obtain valid simulation results. In our work the channel model is based on the VANET channel characterization of Sen and Matolak [31].

For our experiments, packet sizes of 300 bytes are of interest, as explained in section III. When analyzing the packet error rate (PER) in function of signal-to-noise ratio (SNR), similar results were obtained as reported by previous authors [7]-[9]. For short packets of 30 bytes there is almost no impact of mobility. If the packet length is increased to 300 bytes, it can be observed that the useful range diminishes for modulation schemes with a higher rate, and that flooring of the packet error rate significantly impacts reliability over the entire range. In Fig. 4, some of these results are illustrated.

To translate these Matlab model results into link impairment techniques, tables have been constructed for every modulation scheme that indicate the expected PER for a given combination of relative speed difference and SNR. When configuring an experiment, the emulated speed of every node has to be defined. During the experiment, a link impairment software module will process every received packet, and use the packet MAC addresses to identify source and receiver node. The (emulated) relative speed difference can than be calculated, and based on the bitrate and SNR information of the packet header the correct PER for that link can be retrieved from the tables. A random generator with the same probability will then decide to pass the packet to the Linux kernel or to destroy it. 


\section{CONCLUSIONS AND FUTURE WORK}

In this paper it was discussed that a wireless testbed could be a useful complementary tool in VANET research, besides simulation platforms and field operational tests. Based on a breakdown of the VANET scenario, the corresponding requirements were defined. It was shown how the generic indoor wireless testbed IBBT w-iLab.t can be made compliant using only software adjustments. Proposed techniques are an approximation of the IEEE 802.11p standard using .11a hardware, the emulation of mobility based on link impairment, and the use of low transmit power together with manual topology configuration for urban experiments. These techniques are generic, and can be applied on other wireless testbeds. However, the testbed is characterized by some limitations. For the highway setting, it was verified that w-iLab.t can provide densities up to $25 \%$ of the actual maximum VANET density in intense but flowing traffic. Experiments representing urban scenarios are labour intense and limited in topology size. These observations confirm the role of a generic wireless testbed as an additional tool in VANET research, targetting the validation of results obtained in simulators and field operational tests. Future work will focus on optimization techniques focused on the VANET scalability problem. The w-iLab.t testbed will be applied as one of the tools in this research.

\section{REFERENCES}

[1] European Telecommunications Standards Institute, "ETSI TR 102638 Intelligent Transport Systems (ITS); Vehicular communications; Basic set of applications; Definitions", http://www.etsi.org, 2009

[2] S. Eichler, "Performance evaluation of the IEEE 802.11p WAVE communication standard", in Proc. of the IEEE 66th vehicular technology conference, Baltimore, 2007

[3] N. Wisitpongphan, O.K. Tonguz, J.S. Parikh, P. Mudalige, F. Bai, V. Sadekar, "Broadcast storm mitigation techniques in vehicular ad hoc networks", IEEE Wireless Communications, vol. 14, no. 6, pp. 94-94, 2007

[4] D. Jiang, Q. Chen, L. Delgrossi, "Optimal data rate selection for vehicle safety communications", in Proc. of the fifth ACM international workshop on VehiculAr Inter-NETworking (VANET '08), San Fransisco, 2008

[5] K. Bilstrup, E. Uhlemann, E.G. Strm, U. Bilstrup, "On the ability of the 802.11p MAC method and STDMA to support real-time vehicle-tovehicle communication", EURASIP Journal on Wireless Communications and Networking, Vol. 2009, 2009

[6] B. Kloiber, T. Strang, F. de Ponte-Mller, C.R. Garcia, M. Rckl, "An approach for performance analysis of ETSI ITS-G5A MAC for safety applications", in Proc. of the 10th international conference on Intelligen Transport Systems Telecommunications (ITST), Kyoto, 2010

[7] S. Sibecas, C.A. Corral, S. Emami, G. Stratis, "On the suitability of 802.11a/RA for high-mobility DSRC", in Proc. of the IEEE 55th Vehicular Technology Conference, Birmingham, 2002

[8] J. Yin, T. Elbatt, G. Yeung, B.Ryu, S. Habermas, H. Krishnan et al., "Performance Evaluation of Safety Applications over DSRC Vehicular Ad Hoc Networks", in Proc. of the 1st ACM international workshop on Vehicular ad hoc networks (VANET), New York, 2004

[9] I. Ivan, P. Besnier, M. Crussiere, M. Drissi, L. Le Danvic, M. Huard et al., "Physical layer performance analysis of V2V communications in high velocity context", in Proc. of the 9th international conference on Intelligent Transport Systems Telecommunications (ITST), Lille, 2009

[10] W. Vandenberghe, J. Bergs, D. Carels, N. Van den Wijngaert et al., "Impact of introducing road charging on supporting mobile data networks", in Proc. of the 9th international conference on Intelligent Transport Systems Telecommunications (ITST), Lille, 2009

[11] E. Vanhauwaert, S. Verbrugge, W. Vandenberghe, M. Pickavet, P. Demeester, "Realistic cost estimation of an intelligent transportation system roll-out", Journal of the Institute of Telecommunications Professionals, vol 5 , no. 1 , pp. $39-45,2011$
[12] Q. Xu, T. Mak, J. Ko, R. Sengupta, "Medium access control protocol design for vehicle-vehicle safety messages", IEEE Transactions on vehicular technology, vol. 56, no. 2, pp. 449-518, 2007

[13] J.J. Haas, Y.-C. Hu, K.P. Laberteaux, "Real-world VANET security protocol performance", in Proc. of IEEE Global communications conference (Globecom), Honolulu, 2009

[14] European Telecommunications Standards Institute, "ETSI TS 102 6372 Intelligent Transport Systems; Vehicular communications; Basic set of applications; Part 2: specification of cooperative awareness basic service", http://www.etsi.org, 2010

[15] European Telecommunications Standards Institute, "ETSI TS $102637-$ 3 Intelligent Transport Systems; Vehicular communications; Basic set of applications; Part 2: specification of decentralized environment notification basic services", http://www.etsi.org, 2010

[16] C. Zhang, X. Lin, R. Lu, P.-H. Ho, X. Shen, An efficient message authentication scheme for vehicular communications", IEEE Transactions on vehicular technology, vol. 57, no. 6, pp. 3357-3368, 2008

[17] B. Liu, B. Khorashadi, H. Du, D. Ghosal, C.-N. Chuah, M. Zhang, "VGSim: an integrated networking and microscopic vehicular mobility simulation platform", IEEE Communications magazine, vol. 47, no. 5, pp. 134-141, 2009

[18] V. Kumar, R. Bauza, F. Filali, J. Gozalvez, L. Lin, M. Rondinone, "iTETRIS - A large scale integrated simulation platform for V2X communications. Application to real-time traffic management", in Proc. of the 9th international conference on Intelligent Transport Systems Telecommunications (ITST), Lille, 2009

[19] J. Heidemann, N. Bulusu, J. Elson, C. Intanagonwiwat, K.-C. Lan, Y. Xu et al., "Effects of Detail in Wireless Network Simulation", in Proc. Of SCS Communication Networks and Distributed Systems Modeling and Simulation Conference, 2001

[20] D. Cavin, Y. Sasson, A. Schiper, "On the accuracy of MANET simulators", in Proc. of the second ACM international workshop on principles of mobile computing, New York, 2002

[21] D. Kotz, C. Newport, R. S. Gray, J. Liu, Y. Yuan, C. Elliot, "Experimental Evaluation of Wireless Simulation Assumptions", in Proc. of the 7th ACM international symposium on Modeling, analysis and simulation of wireless and mobile systems (MsWiM), Venice, 2004

[22] T. R. Andel, A. Yasinac, "On the credibility of MANET simulations", IEEE Computer, vol. 39, no. 7, pp. 48-54, 2006

[23] C. Cheng, P. Hsiao, H.T. Kung, D. Vla, "Adjacent channel interference in dual-radio 802.11a nodes and its impact on multi-hop networking", in Proc. of IEEE Global communications conference (Globecom), San Fransisco, 2006

[24] H. Stbing, M. Bechler, D. Heussner, T. May, I. Radusch, H. Rechner et al., "simTD: A Car-to-X system architecture for field operational tests", IEEE Communications Magazine, Vol. 48, Issue 5, pp. 148-154, 2010

[25] K. Ramachandran, M. Gruteser, R. Onishi, T. Hikita, "Experimenta analysis of broadcast reliability in dense vehicular networks", IEEE Vehicular Technology Magazine, vol. 2, no. 4, pp. 26-32, 2007

[26] G. Werner-Allen, P. Swieskowski, M. Welsh "Motelab: a Wireless Sensor Network Testbed", in Proc. Of the 4th international symposium on Information processing in sensor networks, Piscataway, 2005

[27] V. Handziski, A. Kpke, A. Willig, A. Wolisz, "TWIST: a Scalable and Reconfigurable Testbed for Wireless Indoor Experiments with Sensor Networks", in Proc. Of the 2nd international workshop on Multi-hop ac hoc networks: from theory to reality, New York, 2006

[28] S. Bouckaert, W. Vandenberghe, B. Jooris, I. Moerman, P. Demeester, "The w-iLab.t Testbed", in Proc. of the 6th international ICST Conference on Testbeds and Research Infrastructures for the development of Networks \& Communities (Tridentcom), Berlin, 2010

[29] Unex, "DCMA-86P2: industrial grade, high power $5.865 .92 \mathrm{GHz}$ wifi mini-PCI module for $802.11 \mathrm{p} / \mathrm{DSRC}$ application, AR5414A-B2B" http://www.unex.com.tw/product/dcma-86p2, last accessed online October 6th 2011

[30] W. Vandenberghe, I. Moerman, P. Demeester, "Approximation of the IEEE $802.11 \mathrm{p}$ standard using commercial off-the-shelf IEEE $802.11 \mathrm{a}$ hardware", in Proc. of the 11th international conference on Intelligent Transport Systems Telecommunications (ITST), Sint-Petersburg, 2011

[31] Sen, I., Matolak, D.W., "Vehicle-Vehicle Channel Models for the 5-GHz Band", IEEE Transactions on Intelligent Transportation Systems, vol.9, no.2, pp.235-245, 2008

[32] J. Kunisch, J. Pamp, "Wideband Car-to-Car Radio Channel Measurements and Model at 5.9 GHz", in Proc. of the 68th IEEE Vehicular Technology Conference, Calgary, 2008 